\title{
Suppression of regucalcin mRNA expression in the hearts of rats administered with free radical compound: The administration- induced death is accelerated in regucalcin transgenic rats
}

\author{
TANIA AKHTER, TAEKO NAKAGAWA, AKIKO KOBAYASHI and MASAYOSHI YAMAGUCHI \\ Laboratory of Endocrinology and Molecular Metabolism, Graduate School of Nutritional \\ Sciences, University of Shizuoka, 52-1 Yada, Suruga-ku, Shizuoka 422-8526, Japan
}

Received October 13, 2006; Accepted November 30, 2006

\begin{abstract}
The expression of regucalcin, a regulatory protein in the intracellular signaling system, in the hearts of rats was investigated. Regucalcin expression was examined using reverse transcription-polymerase chain reaction and Western blot analysis. Regucalcin mRNA and its protein levels in the hearts of male and female rats were significantly decreased with increasing age (50 weeks old) as compared with that of 5-week-old rats. The effect of 1,1-diphenyl-2-picryl-hydrazyl (DPPH), a compound that produces free radical, on regucalcin mRNA expression in the hearts of female rats $(5$ weeks old) was examined. Heart regucalcin mRNA levels were significantly decreased at 60 or $180 \mathrm{~min}$ after a single intraperitoneal administration of DPPH $(0.5 \mathrm{mg} / 100 \mathrm{~g}$ body weight), suggesting that free radical stress has a suppressive effect on the gene expression. Normal (wild) female rats died at approximately $300 \mathrm{~min}$ after a single intraperitoneal administration of DPPH $(0.5 \mathrm{mg} / 100 \mathrm{~g})$, while regucalcin transgenic (TG) female rats died at approximately $150 \mathrm{~min}$ after the administration. Heart regucalcin protein in DPPHadministered rats was greater in regucalcin TG rats than in normal (wild) rats. This study demonstrates that the death of regucalcin TG rats is accelerated after the administration of free radical compound, indicating that overexpression of regucalcin does not have effects as the suppressor for free radical stress and the scavenger for free radical in rats.
\end{abstract}

\section{Introduction}

Regucalcin, which was discovered as a $\mathrm{Ca}^{2+}$-binding protein that does not contain the EF-hand motif as a $\mathrm{Ca}^{2+}$-binding

Correspondence to: Dr Masayoshi Yamaguchi, Laboratory of Endocrinology and Molecular Metabolism, Graduate School of Nutritional Sciences, University of Shizuoka, 52-1 Yada, Suruga-ku, Shizuoka 422-8526, Japan

E-mail: yamaguch@u-shizuoka-ken.ac.jp

Key words: regucalcin, heart, free radical, regucalcin transgenic rats domain $(1,2)$, has been shown to play a multifunctional role as a regulatory protein of intracellular signaling in many cell types (reviewed in refs. 3-6). Rat and human regucalcin genes are localized on chromosome $\mathrm{X}(7,8)$. Regucalcin is greatly expressed in the liver but only to a small extent in the kidney cortex $(9,10)$. In addition, the lower expression of regucalcin is found in rat brain (11), heart (12), and bone tissues (13), suggesting that regucalcin has a role in the regulation of their cellular function.

It has been demonstrated that regucalcin has a role in the maintenance of intracellular $\mathrm{Ca}^{2+}$ homeostasis, the inhibitory regulation of various protein kinases (including $\mathrm{Ca}^{2+}$ / calmodulin-dependent protein kinase, protein kinase $\mathrm{C}$ and thyrosine kinase), protein phosphatases and nitric oxide synthase, which are involved in the intracellular signaling mechanism in many cell types (3-6). Overexpression of regucalcin has suppressive effects on cell proliferation and apoptosis in cultured cells (14-17). Regucalcin localizes in the nucleus, and it suppresses the enhancement of nuclear DNA and RNA synthesis in proliferative cells $(18,19)$. Regucalcin may play a pivotal role in the regulation of cell function.

The role of reguclacin in the heart function has not been fully clarified, although regucalcin mRNA and its protein are expressed in rat heart muscle (12). Recently, it has been shown that regucalcin can activate $\mathrm{Ca}^{2+}$-ATPase, which has a role as a $\mathrm{Ca}^{2+}$ pump, in rat heart microsomes (12) and mitochondria (20), indicating that regucalcin plays a role in the regulation of $\mathrm{Ca}^{2+}$ homeostasis in heart cells. Moreover, regucalcin has been demonstrated to regulate the activities of nitric oxide synthase (21), protein phosphatase (22), and superoxide dismutase (23), which is related to intracellular signaling, in rat heart cytosol. Regucalcin may play a role in the regulation of the heart function.

This study, moreover, was undertaken to determine whether regucalcin mRNA expression is associated with pathophysiologic state including aging and free radical stress in the hearts of rats.

\section{Materials and methods}

Chemicals. 1,1-diphenyl-2-picryl-hydrazyl (DPPH) was purchased from Sigma Chemical Co. (St. Louis, MO, USA). 
Other chemicals were reagent grade from Wako Pure Chemical Industries Ltd. (Osaka, Japan). Reagents used were dissolved in distilled water or ethanol and then passed through an ionexchange resin to remove metal ions.

Animals. Normal male and female rats (Sprague-Dawley, $\mathrm{SD} ; 5$ and 50 weeks old) and regucalcin transgenic (TG) female rats (SD, 5 weeks old) were supplied by Japan SLC (Hamamatsu, Japan). Regucalcin TG rats were generated as described (24). These rats were bled by cardiac puncture with anesthesia of light ethyl ether, and the heart was immediately removed and frozen at $-80^{\circ} \mathrm{C}$ until analysis.

Administration of DPPH. DPPH (25) was dissolved in ethanol (99.5\%). A concentration of DPPH $(0.5$ or $1.0 \mathrm{mg} / 0.5 \mathrm{ml} /$ $100 \mathrm{~g}$ body weight) was intraperitoneally administered to rats, and 10,60 , or 180 min later the animals were bled by cardiac puncture.

Determination of specific mRNA by RT-PCR. Total RNAs were prepared as described previously (26). Aliquots of heart tissue were homogenized in a buffer solution containing $4 \mathrm{M}$ guanidinium thiocyanate, $24 \mathrm{mM}$ sodium citrate $(\mathrm{pH} 7.0)$, $0.5 \%$ sarcosyl, and isoamyl alcohol, and the phases were separated by centrifugation at $10,000 \mathrm{x}$ g for $20 \mathrm{~min}$ at $4^{\circ} \mathrm{C}$. RNA located in the aqueous phase was precipitated with isoprepanol at $-20^{\circ} \mathrm{C}$. RNA precipitates were pelleted by centrifugation, and the pellets were redissolved in diethylpyrocarbonate-treated water.

RT-PCR was performed with a Titan ${ }^{\text {TM }}$ one tube RT-PCR kit (Roche Molecular Biochemicals) as recommended by the supplier to investigate the gene expression of regucalcin or glyceroaldehyde-3-phosphate dehydrogenase (G3PDH). The primers generated were based on the published rat sequences. Primers for regucalcin cDNA were 5'-AGATGAACAAA TCCCAGAT-3' (sense strand, positions 618-696) of cDNA sequence $(27,28)$, and 5'-TCACCCTGCATAGGAATAT-3' (anti-sense strand, positions 906-924). The pair of oligonucleotide primers were designed to amplify a 307-bp sequence from the mRNA of rat regucalcin cDNA. Primers for G3PDH cDNA were 5'-CATGTAGGCCATGAGGTCCACCAC-3' (sense strand) 5'-CATGTAGGCCATGAGGTCCACCAC-3' (anti-sense strand) from the G3PDH amplimer set (Clontech, Palo Alto, CA). RT-PCR was performed using reaction mixture (20 $\mu \mathrm{l}$ ) containing $2 \mu \mathrm{g}$ of total RNAs, supplied RT-PCR buffer, Titam enzyme mix (AMV and Expand ${ }^{\mathrm{TM}}$ High Fidelity), $0.2 \mathrm{mM}$ deoxynucleotide triphosphate, $5 \mathrm{mM}$ dithiothreitol, 5 U RNase inhibitor, $2.5 \mathrm{U}$ TaqDNA polymerase, and $0.3 \mu \mathrm{l}$ primers. Samples were incubated at $50^{\circ} \mathrm{C}$ for $30 \mathrm{~min}$ and at $94^{\circ} \mathrm{C}$ for $2 \mathrm{~min}$, and then amplified for 35 cycles under the following conditions: denaturation for $30 \mathrm{sec}$ at $94^{\circ} \mathrm{C}$, annealing for $30 \mathrm{sec}$ at $60^{\circ} \mathrm{C}$ and extension for $60 \mathrm{sec}$ at $68^{\circ} \mathrm{C}$. The amplified PCR products were separated by electrophoresis on a $1.5 \%$ agarose gel, visualized by ethidium bromide staining and quantitated using a densitometer.

Preparation of heart homogenate. Rats were sacrificed by cardiac puncture, and the heart was perfused with ice-cold $250 \mathrm{mM}$ sucrose solution, immediately cut into small pieces, suspended 1:9 in the homogenization medium containing
$250 \mathrm{mM}$ sucrose, $10 \mathrm{mM}$ 4-(2-hydroxyethyl)-1-piperazineethanesulfonic acid (HEPES), and $1.0 \mathrm{mM}$ ethyleneglycol bis (2-amino-ethylether)-N, N, N', N'-tetraacetic acid (EGTA), $\mathrm{pH} 7.4$, and homogenized in a Potter-Elvehjem homogenizer with a Teflon pestle. The homogenate was centrifuged at $800 \mathrm{x} g$ for $10 \mathrm{~min}$ to remove unbroken cells and cell debris. The resultant supernatant was pooled to use Western blot analysis. The protein concentration was determined using the methods of Lowry et al (29).

Western blot analysis. Aliquots of heart homogenate supernatant (50 $\mu \mathrm{g}$ of protein) from normal rats or regucalcin transgenic rats were mixed with $5 \mathrm{X}$ Laemmli sample buffer, boiled for $5 \mathrm{~min}$, and resolved by $12 \%$ SDS-PAGE (30). The proteins were then transferred onto a polyvinylidene difluoride membrane at $150 \mathrm{~mA}$ for $3 \mathrm{~h} \mathrm{(31).} \mathrm{The} \mathrm{membranes} \mathrm{were}$ incubated with a polyclonal rabbit anti-regucalcin antibody (11), which was diluted 1:2,000 in $10 \mathrm{mM}$ Tris- $\mathrm{HCl}, \mathrm{pH} 8.0$, containing $150 \mathrm{mM} \mathrm{NaCl}, 0.1 \%$ (wt./vol.) Tween-20 (washing buffer), and 5\% (wt./vol.) skim milk for $1 \mathrm{~h}$. The membranes were incubated and washed four times with washing buffer. Then, the membranes were incubated for $1 \mathrm{~h}$ with horseradish peroxidase-linked anti-rabbit $\mathrm{IgG}$, which was diluted 1:5,000 in washing buffer containing 5\% (wt./vol.) skim milk, and again they were washed. Detection of the protein bands was performed using the enhanced chemiluminescent kit following the manufacturer's instructions. The exposure time of the membranes onto film was $10 \mathrm{~min}$. The molecular size of the detecting protein was determined by running the standard protein molecules of known sizes in parallel.

Statistical analysis. Data are expressed as the mean \pm SE. The significance of differences between the values was estimated by Student's t-test or by analysis of variance (ANOVA) for comparing multiple groups. A P-value of $<0.05$ was considered to indicate a statistically significant difference.

\section{Results}

Changes in regucalcin expression in the hearts of rats with different ages. The change in regucalcin mRNA expression in the hearts of male or female rats with different ages was examined using RT-PCR (Fig. 1). Regucalcin mRNA expression in the hearts of male or female rats with increasing age (50 weeks old) was significantly decreased as compared with that of young rats (5 weeks old). However, G3PDH mRNA levels in the heart of male or female rats were not significantly changed with different ages.

The change in regucalcin protein levels in the hearts of male or female rats with different ages using Western blot analysis is shown in Fig. 2. Regucalcin levels were significantly decreased with increasing age of male or female rats.

Change in regucalcin $m R N A$ expression in the hearts of rats administered with DPPH. DPPH is a compound which produces free radical (25). DPPH (1 mg/100 g body weight) was intraperitoneally administrated to female rats (5 weeks old), and 10 or 60 min later the animals were sacrificed 


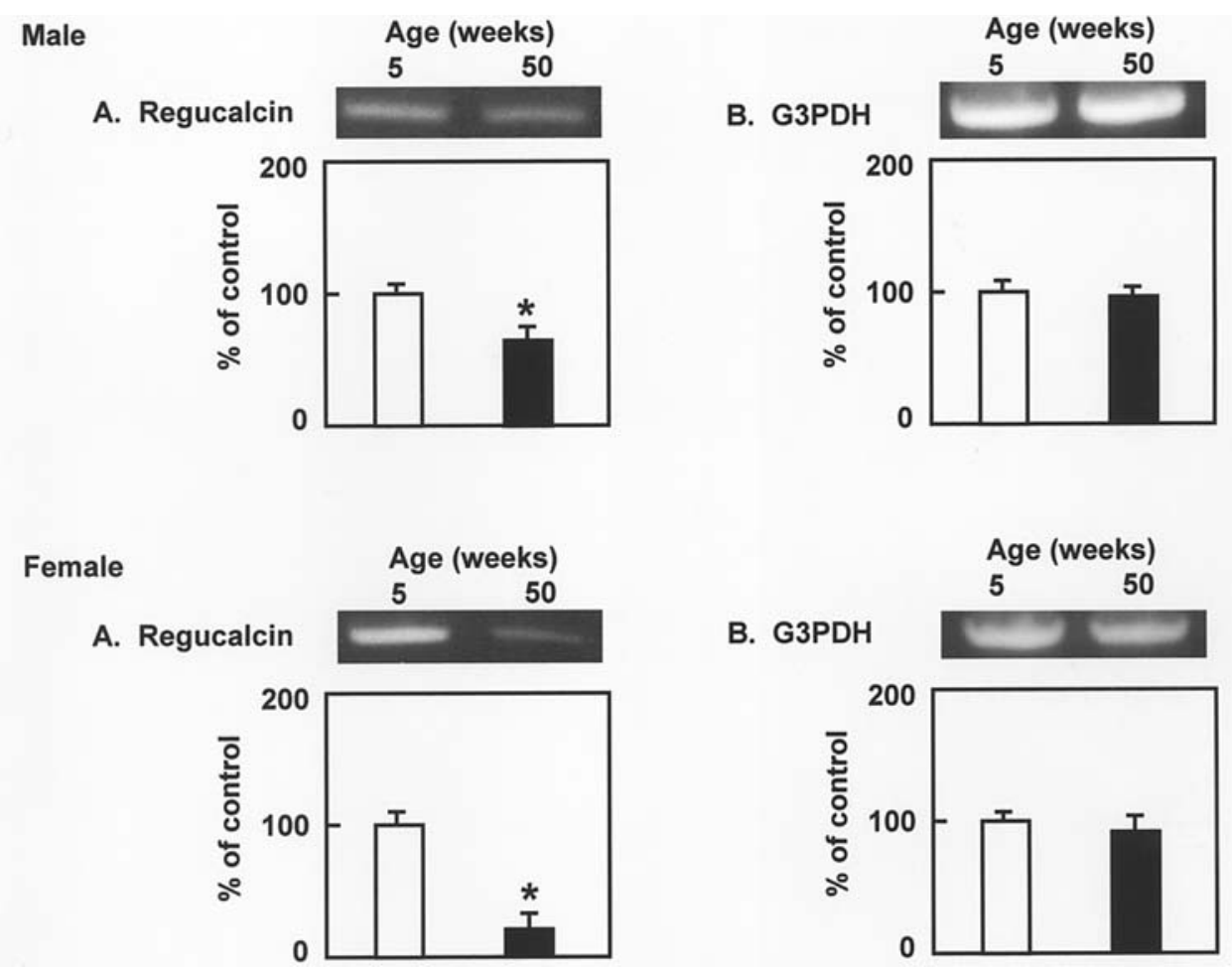

Figure 1. Change in regucalcin mRNA in the hearts of rats with different ages. Heart tissue was obtained from male or female rats (5 or 50 weeks old). Total RNA $(2 \mu \mathrm{g})$ extracted from heart tissue was analyzed by RT-PCR using specific primers of regucalcin (A) or G3PDH (B) cDNAs. The figure shows one of four experiments with separate samples. The densitometric data for regucalcin of G3PDH mRNA levels in the heart tissue of male and female rats (5 or 50 weeks old) were indicated as $\%$ of control ( 5 weeks old) (the mean \pm SE for four experiments). * $\mathrm{p}<0.01$ compared with the value.
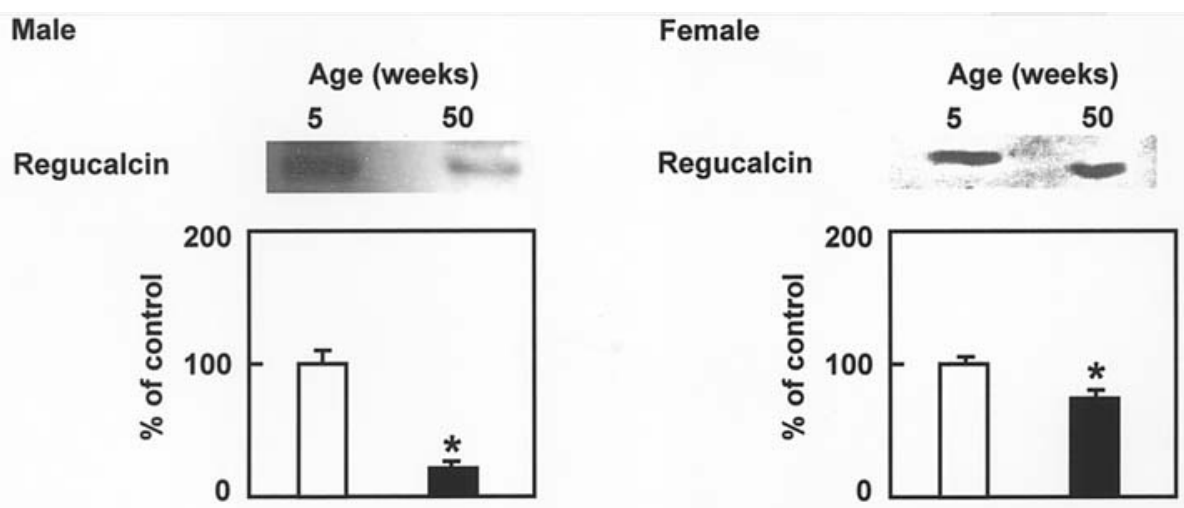

Figure 2. Change in regucalcin protein in the hearts of rats with different ages. Heart tissue was obtained from male or female rats (5 or 50 weeks old). Protein $(50 \mu \mathrm{g})$ extracted from heart tissue was analyzed using Western blot analysis. The figure shows one of four experiments with separate samples. The densitometric data for regucalcin levels in the heart tissues of male and female rats ( 5 or 50 weeks old) were indicated as $\%$ of control ( 5 weeks old) (the mean $\pm \mathrm{SE}$ for four experiments). ${ }^{\mathrm{p}}<0.01$ compared with the value.

(Fig. 3A). Regucalcin mRNA expression in the hearts was significantly decreased at $60 \mathrm{~min}$ after the administration of DPPH. When DPPH $(0.5 \mathrm{mg} / 100 \mathrm{~g})$ was intraperitoneally administered to female rats ( 5 weeks old), regucalcin mRNA levels in the hearts were significantly decreased at 60 and 180 min after the administration (Fig. 3B). Meanwhile, G3PDH mRNA expression was not significantly changed after the administration of DPPH to rats.

Effect of DPPH administration on death of rats. DPPH $(0.5 \mathrm{mg} / 100 \mathrm{~g})$ was intraperitoneally administered to female rats (5 weeks old). Normal (wild) rats died within $\sim 300 \mathrm{~min}$ after the administration of DPPH, while regucalcin transgenic (TG) rats died within $\sim 150 \mathrm{~min}$ (Fig. 4A). Thus the death of regucalcin TG rats was significantly accelerated after the administration of DPPH, which produces free radical. In this case, regucalcin levels in the hearts of regucalcin TG rats were significantly increased as compared with that of normal (or wild) rats (Fig. 4B).

\section{Discussion}

The gene of regucalcin is highly conserved in vertebrate species (28). The regucalcin gene is localized on chromosome $\mathrm{X}$ 


\section{A. DPPH $1 \mathrm{mg} / 100 \mathrm{~g}$}

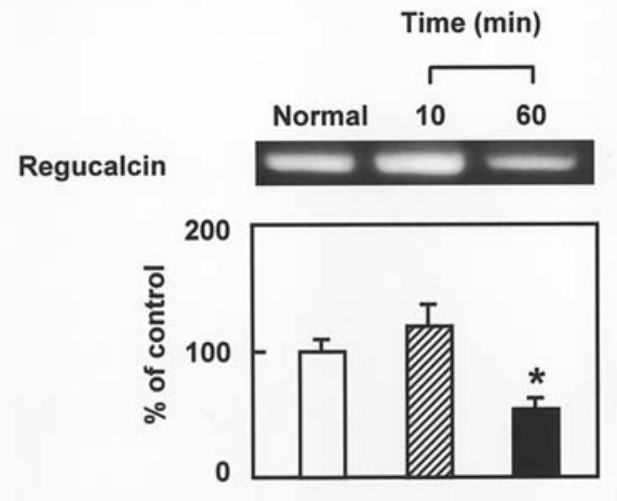

G3PDH

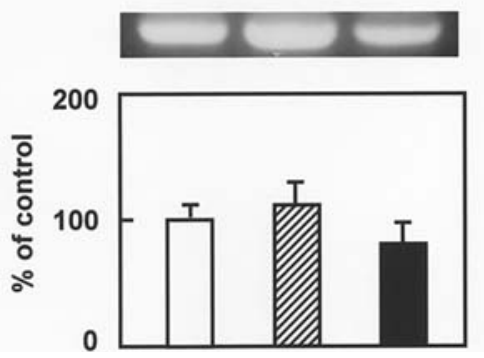

B. DPPH $0.5 \mathrm{mg} / 100 \mathrm{~g}$

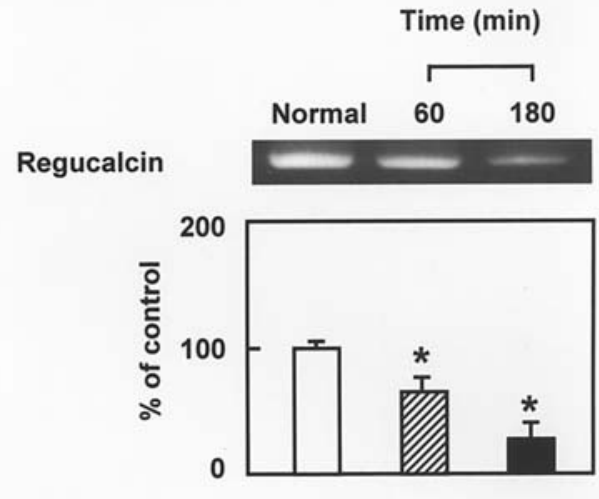

G3PDH

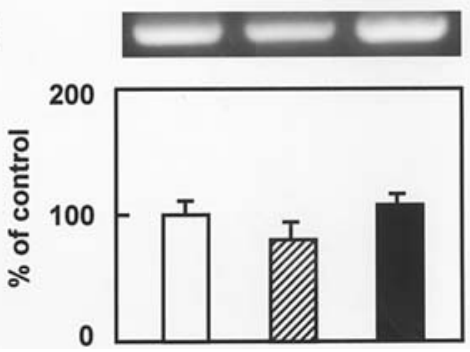

Figure 3. Effect of DPPH administration on regucalcin mRNA expression in the hearts of female rats. (A) The animals (5 weeks old) were sacrificed at 10 or $60 \mathrm{~min}$ after a single intraperitoneal administration of DPPH $(1.0 \mathrm{mg} / 100 \mathrm{~g}$ body weight). (B) The animals (5 weeks old) were sacrificed at $60 \mathrm{or} 180 \mathrm{~min}$ after a single intraperitoneal administration of DPPH $(0.5 \mathrm{mg} / 100 \mathrm{~g})$. The figure shows one of four experiments with separate samples. The densitometric data for regucalcin mRNA levels in the heart tissue were indicated as $\%$ of control (normal) rats (the mean $\pm \mathrm{SE}$ for four experiments). "p $<0.01$ compared with the value.

A.

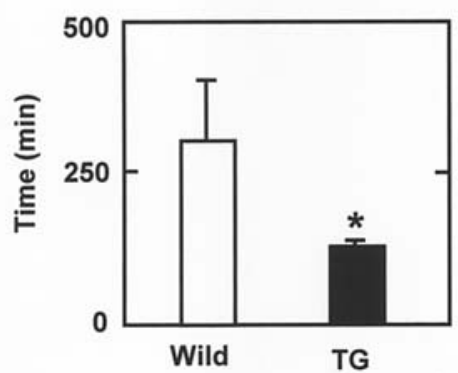

B.

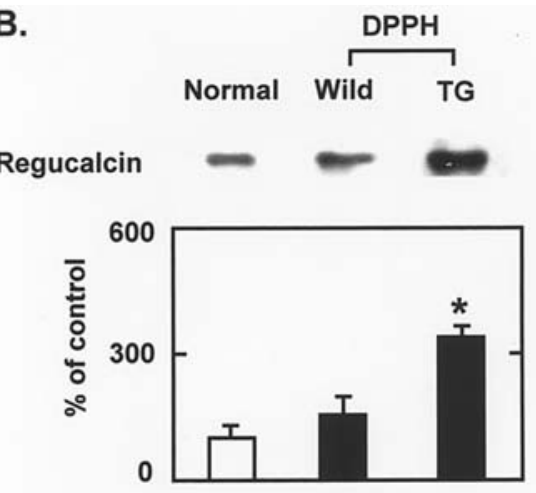

Figure 4. The time to death of normal (wild) rats or regucalcin transgenic rats administered with DPPH and the regucalcin levels in the hearts obtained from $\mathrm{DPPH}$-administered rats. Female wild or regucalcin transgenic rat (5 weeks old) were intraperitoneally administered with DPPH (0.5 mg/100 g body weight). (A) Time was measured when rats died after DPPH administration. Each value is the mean \pm SE for five rats. (B) The hearts were immediately removed when the rats died, and regucalcin protein levels were measured. The figure shows one of four experiments with separate samples. The densitometric data for regucalcin mRNA levels in the heart tissues were indicated as $\%$ of control (normal) rats (the mean $\pm \mathrm{SE}$ for four experiments). ${ }^{*} \mathrm{p}<0.01$ compared with the value.

$(7,8)$. The organization of the rat regucalcin gene consists of seven exons and six introns (34) and several consensus regulatory elements exist in the upstream of the 5'-flanking region of the gene (35). AP-1 (36), NF1-A1 (37) and RGPRP117 (38) have been found to be transcriptional factors for the enhancement of regucalcin gene promoter activity.

Regucalcin is expressed in rat heart (12). This study examined whether regucalcin gene expression alters with pathophysiologic state. Regucalcin mRNA and its protein levels in the heart were found to decrease with increasing age, suggesting a role in the regucalcin of heart cell function with increasing ages.

DPPH is a compound which produces free radical (25). Heart regucalcin mRNA expression was decreased after the administration of DPPH in female rats. It is speculated that free radical stress has a suppressive effect on regucalcin mRNA expression in the hearts of rats. Heart injury with ischemia results from an increase in free radical in the cells (32). The 
suppression of regucalcin gene expression is suggested to have a pathophysiologic role in the development of heart failure with free radical.

Regucalcin has been shown to have a protective effect against the toxicity of free radical using regucalcin transgenic rats that overexpress regucalcin (23). The time to death of female rats after the administration of DPPH was shortened in regucalcin transgenic rats as compared to normal rats. In this case, regucalcin protein levels significantly increased in the hearts of regucalcin transgenic rats in comparison with that of normal rats. This finding indicates that overexpression of regucalcin does not have a protective effect on free radical stress-induced damage in rats, and that the protein does not play a role as the scavenger for free radical.

Bone loss and hyperlipidemia are induced in regucalcin transgenic rats $(13,33)$, suggesting a role of endogenous regucalcin in their metabolism-related regulation. Free radical stress-induced death of rats was found to accelerate in regucalcin transgenic rats. It is possible that regucalcin has a role in the regulation of free radical-involved metabolic function. Regucalcin seems to modulate the effect of free radical stress.

In conclusion, it has been demonstrated that regucalcin mRNA expression in the hearts of rats decreased with increasing age and that free radical stress has a suppressive effect on the gene expression. Moreover, overexpression of regucalcin was found to accelerate free radical stress-induced death of rats.

\section{References}

1. Yamaguchi $\mathrm{M}$ and Yamamoto T: Purification of calcium binding substance from soluble fraction of normal rat liver. Chem Pharm Bull 26: 1915-1918, 1978.

2. Yamaguchi $\mathrm{M}$ and Mori S: Effect of $\mathrm{Ca}^{2+}$ and $\mathrm{Zn}^{2+}$ on $5^{\prime-}$ nucleotidase activity in rat liver plasma membranes: hepatic calcium-binding protein (Regucalcin) reverses the $\mathrm{Ca}^{2+}$ effect. Chem Pharm Bull 36: 321-325, 1988.

3. Yamaguchi $\mathrm{M}$ : A novel $\mathrm{Ca}^{2+}$-binding protein regucalcin and calcium inhibition. Regulatory role in liver cell function. In: Calcium Inhibition. Kohama K (ed). Japan Sci Soc Press, Tokyo and CRC Press, Boca Raton, pp19-41, 1992.

4. Yamaguchi M: Role of regucalcin in calcium signaling. Life Sci 66: 1763-1780, 2000.

5. Yamaguchi M: The role of regucalcin in nuclear regulation of regenerating liver. Biochem Biophys Res Commun 276: 1-6, 2000.

6. Yamaguchi M: Role of regucalcin in maintaining cell homeostasis and function. Int J Mol Med 15: 372-389, 2005.

7. Shimokawa N, Matsuda Y and Yamaguchi M: Genomic cloning and chromosomal assignment of rat regucalcin gene. Mol Cell Biochem 151: 157-163, 1995.

8. Thiselton DL, McDowall J, Brandau O, Ramsere J, d'Esposito F, Bhattacharya SS, Ross MT, Hardcastle AJ and Menindl A: An integrated functionally annotated gene map of the DXS80269ELK1 interval on human Xp 11.3-Xp 11.23: potential hotspot for neurogenetic disorders. Genomics 79: 560-572, 2002.

9. Shimokawa N and Yamaguchi M: Calcium administration stimulates the expression of calcium-binding protein regucalcin mRNA in rat liver. FEBS Lett 305: 151-154, 1992.

10. Yamaguchi $M$ and Isogai M: Tissue concentration of calciumbinding protein regucalcin in rat by enzyme-linked immunoadsorbent assay. Mol Cell Biochem 122: 65-68, 1993.

11. Yamaguchi M, Hamano $\mathrm{T}$ and Misawa $\mathrm{H}$ : Expression of $\mathrm{Ca}^{2+}$ binding protein regucalcin in rat brain neurons: inhibitory effect on protein phosphatase activity. Brain Res Bull 52: 343-348, 2000.

12. Yamaguchi M and Nakajima R: Role of regucalcin as an activator of sarcoplasmic reticulum $\mathrm{Ca}^{2+}$-ATPase activity in rat heart muscle. J Cell Biochem 86: 184-193, 2002.
13. Yamaguchi M, Misawa H, Uchiyama S, Morooka Y and Tsurusaki $Y$ : Role of endogenous regucalcin in bone metabolism: bone loss is induced in regucalcin transgenic rats. Int J Mol Med 10: 377-383, 2002.

14. Misawa H, Inagaki S and Yamaguchi M: Suppression of cell proliferation and deoxyribonucleic acid synthesis in cloned rat hepatoma H4-II-E cells overexpressing regucalcin. J Cell Biochem 84: 143-149, 2002.

15. Nakagawa T, Sawada N and Yamaguchi M: Overexpression of regucalcin suppresses cell proliferation of cloned normal rat kidney proximal tubular epithelial NRK52E cells. Int J Mol Med 16: 637-643, 2005.

16. Izumi $\mathrm{T}$ and Yamaguchi $\mathrm{M}$ : Overexpression of regucalcin suppresses cell death in cloned rat hepatoma H4-II-E cells induced by tumor necrosis factor- $\alpha$ or thapsigargin. J Cell Biochem 92: 296-306, 2004.

17. Nakagawa $\mathrm{T}$ and Yamaguchi $\mathrm{M}$ : Overexpression of regucalcin suppresses apoptotic cell death in cloned normal rat kidney proximal tubular epithelial NRK52E cells: change in apoptosisrelated gene expression. J Cell Biochem 96: 1274-1285, 2005.

18. Tsurusaki Y and Yamaguchi M: Suppressive role of endogenous regucalcin in the enhancement of deoxyribonucleic acid synthesis activity in the nucleus of regenerating rat liver. J Cell Biochem 85: 516-522, 2002.

19. Tsurusaki Y and Yamaguchi M: Role of endogenous regucalcin in nuclear regulation of regenerating rat liver: suppression of the enhanced ribonucleic acid synthesis activity. J Cell Biochem 87: 450-457, 2002.

20. Akhter T, Sawada N and Yamaguchi M: Regucalcin increases $\mathrm{Ca}^{2+}$-ATPase activity in the heart mitochondria of normal and regucalcin transgenic rats. Int J Mol Med 18: 171-176, 2006.

21. Ma ZJ and Yamaguchi M: Suppressive role of endogenous regucalcin in the regulation of nitric oxide synthase activity in heart muscle cytosol of normal and regucalcin transgenic rats. Int J Mol Med 10: 761-766, 2002.

22. Ichikawa E, Tsurusaki Y and Yamaguchi M: Suppressive effect of regucalcin on protein phosphatase activity in the heart cytosol of normal and regucalcin transgenic rats. Int J Mol Med 13: 289-293, 2004.

23. Ichikawa E and Yamaguchi M: Regucalcin increases superoxide dismutase activity in the heart cytosol of normal and regucalcin transgenic rats. Int J Mol Med 14: 691-695, 2004.

24. Yamaguchi M, Morooka Y, Misawa H, Tsurusaki Y and Nakajima R: Role of endogenous regucalcin in transgenic rats: suppression of kidney cortex cytosolic protein phosphatase activity and enhancement of heart muscle microsomal $\mathrm{Ca}^{2+}$ ATPase activity. J Cell Biochem 86: 520-529, 2002.

25. Jin ZQ and Chen $X$ : A single reproducible model of free radicalinjured isolated heart induced by 1,1-diaphyseal-2-picryl-hydrazyl (DPPH). J Pharmacol Toxicol Methods 39: 63-70, 1998.

26. Chomczyshi P and Sacchi N: Single-step method of RNA isolation by acid guanidium thiocyanate-phenol-chloroform extraction. Anal Biochem 162: 156-159, 1987.

27. Shimokawa $\mathrm{N}$ and Yamaguchi $\mathrm{M}$ : Molecular cloning and sequencing of the cDNA coding for a calcium-binding protein regucalcin form rat liver. FEBS Lett 327: 251-255, 1993.

28. Misawa $\mathrm{H}$ and Yamaguchi $\mathrm{M}$ : The gene of $\mathrm{Ca}^{2+}$-binding protein regucalcin is highly conserved in vertebrate species. Int $\mathbf{J} \mathrm{Mol}$ Med 6: 191-196, 2000.

29. Lowry OH, Rosebrogh NH, Farr AL and Randall RF: Protein measurement with the Folin phenol reagent. J Biol Chem 193: 265-273, 1951.

30. Laemmli UK: Cleavage of structural proteins during the assembly of the head of bacteriophage T4. Nature 22: 680-685, 1970.

31. Wessendolf JHM, Ganfinkel S, Zhan X, Brown S and Maciag T: Identification of a nuclear localization sequence within the structure of the human interleukin- $1 \alpha$ precursor. J Biol Chem 268: 22100-22104, 1993.

32. Rajesh KG, Suzuki R, Maeda H, Murio Y and Sasaguri S: 5'$\mathrm{HT}_{2}$ receptor blocker sarpogrelate prevents downregulation of antiapoptotic protein $\mathrm{Bcl}-2$ and protects the heart against ischemiareperfusion injury. Life Sci 79: 1749-1755, 2006.

33. Yamaguchi M, Igarashi A, Uchiyama S and Sawada N: Hyperlipidemia is induced in regucalcin transgenic rats with increasing age. Int J Mol Med 14: 647-651, 2004.

34. Yamaguchi M, Makino R and Shimokawa N: The 5' end sequences and exon organization in the rat regucalcin gene. Mol Cell Biochem 165: 145-150, 1996. 
35. Murata $\mathrm{T}$ and Yamaguchi M: Promoter characterization of the rat gene for $\mathrm{Ca}^{2+}$-binding protein regucalcin. Transcriptional regulation by signaling factors. J Biol Chem 274: 1277-1285, 1999.

36. Murata $\mathrm{T}$ and Yamaguchi $\mathrm{M}$ : $\mathrm{Ca}^{2+}$ administration stimulates the binding of AP-1 factor to the 5'-flanking region of the rat gene for the $\mathrm{Ca}^{2+}$-binding protein regucalcin. Biochem $\mathrm{J} 329$ : 157-183, 1998.
37. Misawa $\mathrm{H}$ and Yamaguchi M: Identification of transcriptional factor in the promoter region of rat regucalcin gene: binding of nuclear factor I-A1 to TTGGC motif. J Cell Biochem 84: 795-802, 2002.

38. Sawada N and Yamaguchi M: Overexpression of RGPR-p117 enhances regucalcin gene expression in cloned normal rat kidney proximal tubular epithelial cells. Int J Mol Med 16: 1049-1055, 2005. 